\title{
The off-shell electromagnetic vertex of the nucleon in chiral perturbation theory
}

\author{
J.W. Bos* \\ National Institute for Nuclear Physics and High Energy \\ Physics Section K (NIKHEF-K) \\ P.O. Box 41882, 1009 DB Amsterdam, The Netherlands \\ and \\ J.H. Koch \\ NIKHEF-K \\ and \\ Institute for Theoretical Physics \\ University of Amsterdam \\ 1018 XE Amsterdam, The Netherlands
}

NIKHEF 93-P1, To be published in Nucl. Phys. A (1993)

\begin{abstract}
We study the electromagnetic vertex of a nucleon in next-to-leading order chiral perturbation theory (CPT). We consider the case where one of the nucleons at the $\gamma \mathrm{NN}$ vertex is off its mass shell. We define relevant measures for the off-shell dependence in the limited kinematical range allowed, and analyze their expansion in the pion mass. The leading nonanalytic contributions are calculated to estimate their size.
\end{abstract}

\section{Introduction}

Many investigations have been carried out recently to examine the electromagnetic interaction of a nucleon not on its mass shell. This was mainly done

*email: jwb@paramount.nikhefk.nikhef.nl 
by using nuclear reactions such as inclusive electron scattering, $\left(\mathrm{e}, \mathrm{e}^{\prime}\right)$, and nucleon knock-out, (e,e'p). However, it also plays a role, under much simpler circumstances, in two-step reactions on a free nucleon, such as pion photo- and electroproduction and Compton scattering. The electromagnetic vertex in these reactions involves a nucleon which does not satisfy the free Dirac equation, and consequently, has a four momentum with $p^{2} \neq M^{2}$. Therefore, it is not correct to use the free nucleon electromagnetic current operator. The operator structure of the off-shell $\gamma \mathrm{NN}$ vertex is more complicated: Its most general form consists of twelve Dirac operators, each multiplied by a form factor which depends on three scalar variables [1]. Clearly, these off-shell form factors cannot directly be obtained from experimental data. There have been some efforts to obtain them using dispersion relations [1, 2, 3. However, because of bad convergence and the absence of the necessary experimental input, this approach was of little use in practice. A microscopic model [4, 5, 6] for the internal nucleon structure is needed to describe the full vertex.

In this paper we examine the general electromagnetic vertex of the nucleon in the framework of chiral perturbation theory (CPT); since we are interested in off-shell effects we must go at least to next-to-leading order, which includes one-loop contributions. A detailed outline of CPT with pions and nucleons, in the relativistic framework, can be found in Refs. [7, 8, 9]. One starts with the most general gauge invariant lagrangian in terms of baryon and meson degrees of freedom, employing the chiral symmetry of the underlying QCD lagrangian in the massless limit. Based on this lagrangian, a perturbation scheme is then developed in which one expands in the (three) momenta of the external particles and simultaneously in the mass of the Goldstone bosons. It has already been applied to pion electro- and photoproduction 10, 11 and Compton scattering at threshold [12, 13]. Certain aspects of the off-shell vertex we study here are already implicitly contained in these low-energy results. We use the relativistic framework; for the heavy baryon limit of CPT we refer e.g. to Ref. 14].

\section{General aspects of the off-shell $\gamma \mathrm{NN}$ vertex}

The general form of the $\gamma \mathrm{NN}$ vertex for the case that both initial and final nucleon are off their mass shell, has been given by Bincer [1]. Using the projection operators

$$
\Lambda^{ \pm}(p)=\frac{ \pm p h+W}{2 W}
$$

with $W=+\sqrt{p^{2}}$, this vertex can be rearranged as

$$
\Gamma_{\mu}^{\mathrm{N}}\left(p^{\prime}, p\right)=-\mathrm{i} e \sum_{\alpha, \beta=-}^{+} \Lambda^{\alpha}\left(p^{\prime}\right)\left[f_{1}^{\alpha \beta} \gamma_{\mu}+f_{2}^{\alpha \beta} \frac{\mathrm{i} \sigma_{\mu \nu} q^{\nu}}{2 M}+f_{3}^{\alpha \beta} q_{\mu}\right] \Lambda^{\beta}(p) .
$$


The initial and final nucleon have four-momentum $p$ and $p^{\prime}$, respectively, and the photon four-momentum is $q=p^{\prime}-p$. The twelve form factors $f_{k}^{\alpha \beta}$ each depend on the three scalar variables, which are usually chosen as $W=\sqrt{p^{2}}$, $W^{\prime}=\sqrt{p^{\prime 2}}$ and $q^{2}$. For simplicity we consider here the situation where the final nucleon is on-shell, $p^{2}=M^{2}$, while the initial nucleon (before absorption of the photon) is off its mass shell. This "half off-shell" vertex can be easily obtained from eq. (2):

$$
\begin{aligned}
\overline{\mathrm{u}}\left(p^{\prime}\right) \Gamma_{\mu}^{\mathrm{N}}\left(p^{\prime}, p\right)= & -\mathrm{i} e \overline{\mathrm{u}}\left(p^{\prime}\right)\left[\left(f_{1}^{++} \gamma_{\mu}+f_{2}^{++} \frac{\mathrm{i} \sigma_{\mu \nu} q^{\nu}}{2 M}+f_{3}^{++} q_{\mu}\right) \Lambda^{+}(p)\right. \\
& \left.+\left(f_{1}^{+-} \gamma_{\mu}+f_{2}^{+-} \frac{\mathrm{i} \sigma_{\mu \nu} q^{\nu}}{2 M}+f_{3}^{+-} q_{\mu}\right) \Lambda^{-}(p)\right],
\end{aligned}
$$

where $f_{i}^{+ \pm}=f_{i}^{+ \pm}\left(q^{2}, W, M\right)$. We will from now on work with the reducible vertex operator, which includes self-energy insertions into the external nucleon legs. Taking the final nucleon on-shell, we need to insert the self-energy into the initial nucleon leg only.

In order to show the connection between the general form factors in eqs. (2) and (3) and the usual free form factors, we evaluate $\Gamma_{\mu}^{\mathrm{N}}\left(p^{\prime}, p\right)$ between free onshell nucleon spinors. Using eq. (3) one finds for the Dirac form factor [1]

$$
F_{1}\left(q^{2}\right)=f_{1}^{++}\left(q^{2}, M, M\right),
$$

and for the Pauli form factor

$$
F_{2}\left(q^{2}\right)=f_{2}^{++}\left(q^{2}, M, M\right) .
$$

Even though they do not appear in the free current, we will also use the analogously defined functions

$$
\mathcal{F}_{1}\left(q^{2}\right)=f_{1}^{+-}\left(q^{2}, M, M\right),
$$

and

$$
\mathcal{F}_{2}\left(q^{2}\right)=f_{2}^{+-}\left(q^{2}, M, M\right) .
$$

Below, we will only discuss the four half off-shell form factors $f_{1}^{+ \pm}$and $f_{2}^{+ \pm}$, since $f_{3}^{+ \pm}$is directly related to $f_{1}^{+ \pm}$through the Ward-Takahashi identity [1].

\section{$3 \quad$ The $\gamma$ NN vertex in $\mathrm{CPT}$}

We will calculate the electromagnetic interaction term in next-to-leading order CPT, i.e. up to order $\mathcal{O}\left(E^{3}\right)$, where $E$ is a generic notation for the fourmomentum of the external photon, the three-momenta of the external nucleons, and the meson mass [7]. For this purpose we need the CPT lagrangian up to order $\mathcal{O}\left(E^{3}\right)$, which reads

$$
\mathcal{L}=\mathcal{L}_{1}+\mathcal{L}_{2},
$$


Figure 1: Loop diagrams contributing to the half off-shell electromagnetic vertex.

where the first part, $\mathcal{L}_{1}$, is the standard nonlinear $\sigma$-model lagrangian. We restrict ourselves here to the $\mathrm{SU}(2)$ flavor group and the isospin symmetric limit where $m_{\mathrm{u}}=m_{\mathrm{d}}=m$, explicitly only considering pions and nucleons. In Refs. [7] and [8] the second part of the lagrangian eq. (8), $\mathcal{L}_{2}$, is given sufficient for the situation where one considers on-shell matrix elements of the $\gamma \mathrm{NN}$ vertex. For off-shell particles, however, one needs to take into account more terms. For our situation the necessary expression is, in the notation of Ref [7],

$$
\mathcal{L}_{2}=\mathcal{L}_{2}^{(0)}+\mathcal{L}_{2}^{(2)}+\mathcal{L}_{2}^{(3)}
$$

where

$$
\begin{aligned}
\mathcal{L}_{2}^{(0)}= & \Delta M \frac{M^{2}}{F^{2}} \bar{\psi} \psi \\
\mathcal{L}_{2}^{(2)}= & c_{1} \frac{4 M_{\pi}^{2} M}{F^{2}} \bar{\psi} \psi+c_{6} \frac{M}{F^{2}} \bar{\psi} \sigma^{\mu \nu} f_{\mu \nu}^{+} \psi+n_{1} \frac{M}{F^{2}} \bar{\psi}(\mathrm{i} \not D-M)^{2} \psi \\
\mathcal{L}_{2}^{(3)}= & b_{9} \frac{1}{F^{2}} \bar{\psi} \gamma^{\mu}\left[D^{\nu}, f_{\mu \nu}^{+}\right] \psi+b_{12} \frac{4 M_{\pi}^{2}}{F^{2}} \bar{\psi}(\mathrm{i} \not D-M) \psi \\
& +n_{2} \frac{1}{F^{2}} \bar{\psi}\left\{(\mathrm{i} \not D-M), \sigma^{\mu \nu} f_{\mu \nu}^{+}\right\} \psi+n_{3} \frac{1}{F^{2}} \bar{\psi}(\mathrm{i} \not D-M)^{3} \psi
\end{aligned}
$$

Here $D_{\mu}$ is the covariant derivative and $f_{\mu \nu}^{+}$is the electromagnetic field strength tensor. The "low-energy constants" $\Delta M, c_{1}, \ldots, n_{3}$ in $\mathcal{L}_{2}$ are parameters needed for the renormalization of the ultra-violet divergences. The constants $n_{1}$, $n_{2}$, and $n_{3}$ in eqs. (11) and (12) only appear in the half off-shell electromagnetic vertex, which is not a direct observable. In a physical amplitude where this half off-shell vertex plays a role, e.g. Compton scattering on a free nucleon, such terms are included through counterterms for the entire amplitude.

For the evaluation of the $\gamma \mathrm{NN}$ vertex in next-to-leading order we need diagrams from $\mathcal{L}_{1}$ up to the one-loop level (shown in fig. 1) and from $\mathcal{L}_{2}$ only on the tree-level. Applying the standard Feynman rules, and using the renormalization prescription of dimensional regularization, one obtains for example for diagram (1d):

$$
\begin{aligned}
\Gamma_{\mu}^{\mathrm{d}}= & M_{\mathrm{ref}}^{4-n} \int \frac{d^{n} k}{(2 \pi)^{n}} \frac{g_{\mathrm{A}}}{2 F}(-\not k-\not q) \gamma_{5} \tau_{i} \Delta(k+q) \\
& \times\left[-e \epsilon^{i j 3}(2 k+q)_{\mu}\right] \Delta(k) S(p-k) \frac{g_{\mathrm{A}}}{2 F} \not k \cdot \gamma_{5} \tau_{j} .
\end{aligned}
$$

Here $S$ and $\Delta$ are the bare nucleon and pion propagator, respectively. As this example shows, the loop diagrams diverge for $n=4$. Finite results are obtained by using the counterterms from $\mathcal{L}_{2}$, eq. (9). The constants $n_{1}$ and $n_{3}$ are needed to renormalize the off-shell irreducible $\gamma \mathrm{NN}$ vertex and the nucleon 
self-energy. However, in the reducible vertex we consider here they drop out. We will later discuss the relevance of the constant $n_{2}$ for our situation; the other counterterms, which enter when renormalizing the on-shell (observable) properties, were already discussed in Ref. [7].

Let us now discuss to what extent we can obtain the half off-shell electromagnetic form factors in eq. ( 3) with this next-to-leading order calculation. Since $A_{\mu}=\mathcal{O}(E)$, the $\gamma \mathrm{NN}$ vertex can be evaluated up to $\mathcal{O}\left(E^{2}\right)$, yielding a total interaction term of $\mathcal{O}\left(E^{3}\right)$. In this expansion scheme the operators in eq. (3) are counted as

$$
\Lambda^{+}(p)=\mathcal{O}(1) ; \Lambda^{-}(p)=\mathcal{O}(E) ; q^{\nu}=\mathcal{O}(E) .
$$

It implies that we can obtain $f_{1}^{++}$up to terms of $\mathcal{O}\left(E^{2}\right), f_{1}^{+-}$and $f_{2}^{++}$up to terms of $\mathcal{O}(E)$, and $f_{2}^{+-}$up to a constant. Since for the scalar variables one has

$$
W-M=\mathcal{O}(E) ; q^{2}=\mathcal{O}\left(E^{2}\right),
$$

we may only use the calculation in a limited kinematical region, where $W$ is close to $M$ and $q^{2}$ is small. As we will see, however, the expansion in $E$ also contains nonanalytic terms in the quark mass (or in the pion mass, $M_{\pi}$, since $M_{\pi}^{2} \sim m$ ). It is generally assumed that they can uniquely be obtained from a one-loop CPT calculation [7, 9]. In this paper, we will focus on the leading singular terms in the chiral limit, $M_{\pi} \rightarrow 0$. Since the actual pion mass is small these contributions can be expected to be the dominant.

Expanding around the photon point, one has for a given form factor

$$
f\left(q^{2}, W, M\right)=f(0, W, M)+\left.\frac{\partial}{\partial q^{2}} f\left(q^{2}, W, M\right)\right|_{q^{2}=0}+\ldots .
$$

For $f_{1}^{++}$and $f_{1}^{+-}$, the first term in such an expansion is defined by the nucleon charge, independent of $W$ [i]. The $W$ dependence will first show up in the second term, and we will therefore below consider

$$
\left.6 \frac{\partial^{2}}{\partial W \partial q^{2}} f_{1}^{+ \pm}\left(q^{2}, W, M\right)\right|_{W=M, q^{2}=0} .
$$

We have included the factor 6 since for $f_{1}^{++}$the second term in the right hand side of eq. (16) is related to the mean square radius of the nucleon according to

$$
\left\langle r^{2}\right\rangle=\left.6 \frac{d}{d q^{2}} F_{1}\left(q^{2}\right)\right|_{q^{2}=0} .
$$

Therefore, the quantity in eq. (17) for $f_{1}^{++}$can be seen as the change of the mean square radius with $W$.

Since for $f_{2}^{++}$and $f_{2}^{+-}$already the first term of the expansion eq. (16) is $W$ dependent 相, 田, we will consider the leading off-shell variation

$$
\left.\frac{\partial}{\partial W} f_{2}^{+ \pm}(0, W, M)\right|_{W=M} \text {. }
$$


If there are nonanalytic terms in the quantities defined in eqs. (17) and (19) then they are determined by a one-loop calculation. According to the above power counting rules, the analytic terms for these properties for $f_{1}^{++}, f_{1}^{+-}$and $f_{2}^{+-}$fall outside the scope of this paper.

\subsection{The four form factors at $W=M$}

Before discussing the above off-shell properties, we first will examine the four form factors defined in eqs. (14) to ( 7 ), i.e. $F_{1}, \mathcal{F}_{1}, F_{2}$, and $\mathcal{F}_{2}$.

1. The relevant feature for the charge form factor $F_{1}$, for low $q^{2}$, is the mean square radius defined in eq. (18). Its isovector part has already been calculated by Gasser et al [7]. They found

$$
\left\langle r^{2}\right\rangle^{\mathrm{V}}=a_{-1} \ln x+a_{0}+\mathcal{O}(\sqrt{x})
$$

where $x=M_{\pi}^{2} / M^{2}$ is linear in the quark mass. CPT fixes the coefficient of the leading nonanalytic contribution,

$$
a_{-1}=-\frac{1}{16 \pi^{2}} \frac{1}{F^{2}}\left(1+5 g_{\mathrm{A}}^{2}\right) .
$$

However, the next term in eq. 201, $a_{0}$, contains the low-energy parameter $b_{9}^{\mathrm{V}}$, appearing in the isovector part of eq. (12). Numerically, the leading term in eq. (20) gives an isovector mean radius of $1.0 \mathrm{fm}$, while the experimental value is $0.8 \mathrm{fm}$. The constant $a_{0}$ can be fitted to exactly arrive at the experimental value (which amounts to fixing $b_{9}^{\mathrm{V}}$ ).

We find for the isoscalar part of the mean square radius

$$
\left\langle r^{2}\right\rangle^{\mathrm{S}}=b_{0}+\mathcal{O}(\sqrt{x})
$$

where $b_{0}$ is determined by the isoscalar constant $b_{9}^{\mathrm{S}}$ in eq. (12). Therefore, in contrast to $\left\langle r^{2}\right\rangle^{\mathrm{V}}$, the leading part of $\left\langle r^{2}\right\rangle^{\mathrm{S}}$ is not a singular term. The constant $b_{0}$ can be used to fit the experimental value, which fixes $b_{9}^{\mathrm{S}}$.

2. The slope of the isovector form factor $\mathcal{F}_{1}^{\mathrm{V}}$ at the photon point is given by

$$
\left.6 \frac{d}{d q^{2}} \mathcal{F}_{1}^{\mathrm{V}}\left(q^{2}\right)\right|_{q^{2}=0}=c_{-1} \ln x+\mathcal{O}(1)
$$

where the coefficient of the leading singular term is

$$
c_{-1}=-\frac{1}{16 \pi^{2}} \frac{1}{F^{2}}\left(1-g_{\mathrm{A}}^{2}\right)
$$


Since $g_{\mathrm{A}}=1.25$, this is a much smaller leading coefficient than $a_{-1}$ in eq. (20), indicating that $\mathcal{F}_{1}^{\mathrm{V}}\left(q^{2}\right)$ has a weaker $q^{2}$ dependence than $F_{1}^{V}$ in the chiral limit. Consequently, the higher order terms in eq. (23) can be important, but are outside the scope of our $\mathcal{O}\left(E^{2}\right)$ calculation of the vertex.

The slope of the isoscalar form factor $\mathcal{F}_{1}^{\mathrm{S}}\left(q^{2}\right)$ has no singular leading contributions. From the operator structure one can see that an analytic contribution (starting with a constant) will lead at least to an $\mathcal{O}\left(E^{3}\right)$ contribution to the vertex. Again, this is outside the scope of our calculation.

3. More terms in the expansion in $x$ of the isovector anomalous magnetic moment, $F_{2}^{\mathrm{V}}(0)$, can be obtained with our calculation. The expansion is given by [7]

$$
F_{2}^{\mathrm{V}}(0)=8 c_{6}^{\mathrm{V}} \frac{M^{2}}{F^{2}}+\frac{g_{\AA}^{2}}{16 \pi^{2}} \frac{M^{2}}{F^{2}}[5-4 \pi \sqrt{x}-7 x \ln x+\mathcal{O}(x)],
$$

while we find for the isoscalar part

$$
F_{2}^{\mathrm{S}}(0)=8 c_{6}^{\mathrm{S}} \frac{M^{2}}{F^{2}}-\frac{g_{\mathrm{A}}^{2}}{16 \pi^{2}} \frac{M^{2}}{F^{2}}[3+3 x \ln x+\mathcal{O}(x)] .
$$

Thus, both the isoscalar and isovector anomalous magnetic moment remain finite in the chiral limit. Their leading contribution, the constant term in eqs. (25) and (26), already contain the free constants $c_{6}^{\mathrm{S}}$ and $c_{6}^{\mathrm{V}}$, which one can use to fit the experimental values.

4. At the photon point the +- form factors $\mathcal{F}_{2}^{\mathrm{S}}$ and $\mathcal{F}_{2}^{\mathrm{V}}$ are simply given by

$$
\mathcal{F}_{2}^{\mathrm{S} / \mathrm{V}}(0)=8 c_{6}^{\mathrm{S} / \mathrm{V}}-8 n_{2}^{\mathrm{S} / \mathrm{V}}+\mathcal{O}(x),
$$

i.e. their leading contributions are determined by the low-energy constants from $\mathcal{L}_{2}$. In contrast to the low-energy constants $c_{6}^{\mathrm{S}}$ and $c_{6}^{\mathrm{V}}$, which enter the anomalous magnetic moments eqs. (25) and (26) and can be determined by fitting this property, $n_{2}^{\mathrm{S}}$ and $n_{2}^{\mathrm{V}}$ in eq. (27) do not enter the on-shell nucleon matrix elements. Stated differently, to obtain the leading contributions to $\mathcal{F}_{2}^{\mathrm{S} / \mathrm{V}}(0)$ we need more information than provided by chiral symmetry and on-shell nucleon properties alone.

Note that an unsubtracted dispersion relation would lead to [2]

$$
F_{2}^{\mathrm{S} / \mathrm{V}}(0) \approx \frac{1}{2} \sqrt{x} \mathcal{F}_{2}^{\mathrm{S} / \mathrm{V}}(0),
$$

which is not satisfied as can be seen by comparing the powers of $\sqrt{x}$ in eq. (27) and in eqs. (25) and (26). Nyman had already questioned the validity of this 
approach in his study of p-p bremsstrahlung 15]; the above provides another reason. such an approach would fail.

Therefore, for the two quantities $\mathcal{F}_{1}$ and $\mathcal{F}_{2}$, which do not occur in the free on-shell current, we cannot extract any definitive information. While the leading singular term for $\mathcal{F}_{1}^{\mathrm{V}}$ is determined, it is unlikely that it will be numerically significant.

\subsection{Off-shell dependence of the form factors}

We will now turn to the off-shell behavior of the form factors, in particular the quantities defined by eqs. (17) and (19).

1. For the isovector form factors $f_{1}^{++, \mathrm{V}}$ and $f_{1}^{+-, \mathrm{V}}$ we find at $q^{2}=0$ and $W=M$

$$
6 \frac{\partial^{2}}{\partial W \partial q^{2}} f_{1}^{++, \mathrm{V}}=\frac{5}{32 \pi} \frac{g_{\mathrm{A}}^{2}}{F^{2} M} \frac{1}{\sqrt{x}}+\mathcal{O}(\ln x),
$$

and

$$
6 \frac{\partial^{2}}{\partial W \partial q^{2}} f_{1}^{+-, \mathrm{V}}=-\frac{1}{16 \pi} \frac{g_{\mathrm{A}}^{2}}{F^{2} M} \frac{1}{\sqrt{x}}+\mathcal{O}(\ln x) .
$$

Therefore, in the chiral limit both quantities diverge. From eq. (29) we can see that the isovector mean square radius of a nucleon with $W<M$ is smaller than that of a free one. This is in qualitative agreement with

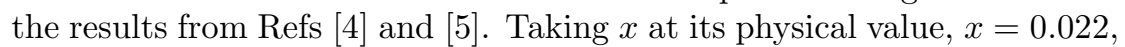
and retaining only the leading terms in eq. (29), we find in CPT that the free $(W=940 \mathrm{MeV})$ mean square radius of $0.6 \mathrm{fm}^{2}$ is reduced by about $0.1 \mathrm{fm}^{2}$ if we use eq. (29) to extrapolate linearly to $W=890 \mathrm{MeV}$. The above illustrates that the "radius of an off-shell nucleon" has not a fixed value, but is a dynamic property depending on the kinematical variables (in our case, where the nucleon is half off-shell, this variable is $W$ ). Comparing eqs. (29) and (30) indicates that the $W$ dependence of $f_{1}^{+-, \mathrm{V}}$ is smaller than that of $f_{1}^{++, \mathrm{V}}$. It is interesting that the slope of $f_{1}^{+-, V}$ near the photon point increases for $W<M$, in contrast to that of $f_{1}^{++, \mathrm{V}}$.

2. The singular terms for the isoscalar form factors $f_{1}^{++, \mathrm{S}}$ and $f_{1}^{+-, \mathrm{S}}$ vanish:

$$
6 \frac{\partial^{2}}{\partial W \partial q^{2}} f_{1}^{+ \pm, S}=\mathcal{O}(1)
$$

Their expansions start with a $\mathcal{O}(1)$ term, indicating a weaker off-shell dependence than the isovector parts. As already discussed, these analytic contributions (which are now the leading one) cannot be obtained from our calculation. 
3. The off-shell variation of the isovector form factors $f_{2}^{++, \mathrm{V}}$ and $f_{2}^{+-, \mathrm{V}}$ at $q^{2}=0$ and $W=M$ reads

$$
\frac{\partial}{\partial W} f_{2}^{++, \mathrm{V}}=d_{-1} \ln x+d_{0}+\mathcal{O}(\sqrt{x})
$$

and

$$
\frac{\partial}{\partial W} f_{2}^{+-, \mathrm{V}}=-d_{-1} \ln x+\mathcal{O}(1)
$$

respectively, where

$$
d_{-1}=-\frac{g_{\mathrm{A}}^{2}}{8 \pi^{2}} \frac{M}{F^{2}},
$$

and $d_{0}$ is a constant containing $n_{2}^{\mathrm{V}}$. Note that, according to eq. (27), $n_{2}^{\mathrm{V}}$ also determines the on-shell limit of $f_{2}^{+-, \mathrm{V}}$. In contrast to their values at the photon point, given in eqs. (25) and (27), the dependence of $f_{2}^{++, \mathrm{V}}$ and $f_{2}^{+-, \mathrm{V}}$ on $W$ are fixed, in leading order. The leading singular terms in eqs. (32) and (33) are of equal magnitude but have opposite sign. Retaining only the leading contribution in eq. (32), we find that if we extrapolate to $W=890 \mathrm{MeV}$ the off-shell isovector anomalous magnetic moment decreases by 0.4 , about $10 \%$ of the on-shell value.

4. Again, the derivatives of the isoscalar form factors $f_{2}^{++, S}$ and $f_{2}^{+-, S}$ with respect to $W$ are not singular in the chiral limit. Since the vertex operators multiplying $f_{2}^{+-, S}$ in the vertex are already of order $\mathcal{O}\left(E^{2}\right)$ the absence of a singular term means that we cannot make a prediction for this term. The operators for $f_{2}^{++, S}$ are of order $\mathcal{O}(E)$. The expansion of the derivative starts off with a constant term containing $n_{2}^{\mathrm{S}}$, not further restricted by chiral symmetry. As already discussed in connection with $\mathcal{F}_{2}^{\mathrm{S}}(0)$, eq. (27), this constant is not constrained by on-shell properties of the nucleon. Therefore, no prediction result for the off-shell variation of both isoscalar form factors.

In the above, we have only been able to determine off-shell effects through nonanalytic terms in the isovector part of the vertex. These effects are entirely due to the contribution from the loop diagram (d) in fig. 1, given by eq. (13), which is purely isovector.

\section{Summary}

A way to address the electromagnetic interaction of an off-shell nucleon in a dynamical fashion is chiral perturbation theory. It is a general approach based 
on the fundamental QCD symmetries. We are interested in the off-shell behavior of the reducible electromagnetic vertex. This means in CPT that one has to expand the lagrangian at least to $\mathcal{O}\left(E^{3}\right)$, where $E$ is a generic nucleon threemomentum, photon four-momentum or a meson mass. We restrict ourselves to an $\mathcal{O}\left(E^{3}\right)$ calculation. First, this means that one has to take into account oneloop contributions due to iterating the lowest order term in the lagrangian. It is generally assumed that the one-loop diagrams yield all the non-analytic terms in the expansion due to the expansion in the pion mass. Since the pion mass is small, one can expect that these nonanalytic contributions provide a relevant part of the full expansion. Second, the higher order terms (up to $\mathcal{O}\left(E^{3}\right)$ ) in the lagrangian also must be taken into account, but only at the tree level. There are also contributions in this order from higher loops; they are effectively contained in the parameters of the lagrangian, which are not predicted by the theory. The parameters that appear in the on-shell case can be used to fit the free nucleon properties.

In an expansion to this order the range of kinematical variables of the vertex is restricted. In practice, this means that e.g. only the slope of the form factors can be studied. We have, for simplicity, studied the "half off-shell" electromagnetic vertex, i.e. the situation where only one of the nucleons at the vertex is off its mass shell. The corresponding reducible form factors $f_{1}^{++}$and $f_{1}^{+-}$ are restricted to the nucleon charge at the photon point, independent of $W$. Therefore, off-shell features in $f_{1}^{++}$and $f_{1}^{+-}$first show up in the slope of these form factors, e.g. for $f_{1}^{++}$as the $W$ dependence of the mean square radius. Our calculation shows that in general only the isovector off-shell form factors contain singular contributions which can be determined already at the one-loop level. For $f_{1}^{++, \mathrm{V}}$ this contribution indicates a reduction of the free isovector mean square radius when $W<M$; for example a decrease of about $15 \%$ at $W=890 \mathrm{MeV}$. For $f_{1}^{+-, \mathrm{V}}$, it increases for $W<M$, but this effect is much smaller.

For the half off-shell form factors $f_{2}^{++}$and $f_{2}^{+-}$a dependence on the invariant mass $W$ already occurs at the photon point. Decreasing $W$ from the free mass to $890 \mathrm{MeV}$ we obtain from the leading terms a decrease of $f_{2}^{++, \mathrm{V}}$ at the photon point, while $f_{2}^{+-, \mathrm{V}}$ increases by that amount. For the half off-shell isoscalar derivatives one does not have a leading singular contribution. The analytic terms depend on low-energy constants which only enter in the electromagnetic vertex involving an off-shell nucleon, and therefore cannot be determined from on-shell properties.

In our approach, we have worked in the nonstrange sector. Furthermore, only the pion, the lightest meson appears, i.e. only the "soft" degrees of freedom are explicitly taken into account. The "hard" physics due to heavier mesons appears through the choice of the renormalization constants as was argued, e.g. in connection with the renormalization constant $c_{6}$ [9]. In this paper, we have focussed on the leading singular terms in the half off-shell form factors. 
The exact value of the quantities considered depends on the size of the higherorder contributions. However, our calculations were able to show qualitative features of the electromagnetic properties of a nucleon not on its mass shell. Our results once again show that these properties, such as the "radius of an off-shell nucleon," are not fixed properties, but depend on the kinematical parameters and dynamical circumstances.

\section{Acknowledgement}

We would like to thank S. Scherer for useful criticism. This work was made possible by financial support from the Foundation for Fundamental Research on Matter (FOM) and the Netherlands Organization for Scientific Research (NWO).

\section{References}

[1] A.M. Bincer, Phys. Rev. 118, 855 (1960).

[2] E.M. Nyman, Nucl. Phys. A154, 97 (1970).

[3] M.G. Hare, Annals of Physics 74, 595 (1972).

[4] H.W.L. Naus and J.H. Koch, Phys. Rev. C36, 2459 (1987).

[5] P.C. Tiemeijer and J.A. Tjon, Phys. Rev. C42, 599 (1990).

[6] X. Song et al, Zeit. Phys. A341, 275 (1992).

[7] J. Gasser, M.E. Sainio, and A. S̆varc, Nucl. Phys. B307, 779 (1988).

[8] A. Krause, Helv. Phys. Acta 63, 1 (1990).

[9] U.-G. Meißner, Int. Journ. Mod. Phys. E1, 561 (1992).

[10] V. Bernard, N. Kaiser, J. Gasser, and U.-G. Meißner, Phys. Lett. B268, 291 (1991).

[11] V. Bernard, N. Kaiser, and U.-G. Meißner, Phys. Lett. B282, 448 (1992).

[12] V. Bernard, N. Kaiser, and U.-G. Meißner, Nucl. Phys. B373, 346 (1992).

[13] M.N. Butler and M.J. Savage, Phys. Lett. B294, 369 (1992).

[14] E. Jenkins and A. Manohar, in Effective field theories of the standard model, ed. U.-G. Meißner (World Scientific, Singapore, 1992).

[15] E.M. Nyman, Nucl. Phys. A160, 517 (1971). 